\title{
Use of the FRAX calculator with and without bone mineral density in Greek women
}

\author{
Ioannis Ilias, Filio Spanoudi, Eftychia Koukkou, Stamatina C. Nikopoulou \\ Department of Endocrinology, Diabetes and Metabolism, E. Venizelou Hospital, Athens, Greece
}

The creators of the FRAX fracture risk calculator for countries with no national reference values (such as Greece) suggest the use of FRAX from a neighboring country. ${ }^{1,2}$ For Greeks (considered to run a high risk for osteoporotic fractures), the Italian FRAX is the suggested choice. ${ }^{2}$ The correlation of FRAX results with and without the use of femoral neck T-scores assessed by DXA is considered to be adequate. The aim of our study was to assess the correspondence of 10 -year femoral neck fracture risk with and without the inclusion of femoral neck T-scores implementing the Italian FRAX in Greek women and to evaluate, albeit indirectly, its validity.

We studied 88 consecutive women outpatients (mean age \pm SD: $58 \pm 8$ years) with 0 -3 FRAX risk factors. All had neck femoral bone mineral density (BMD) assessed by DXA and received only calcium supplements \pm vitamin D3 (500 mg/day to1200 mg/ day $\pm 400 \mathrm{IU} /$ day to $1200 \mathrm{IU} /$ day, respectively); all were bisphosphonate-naive. We calculated the 10-year femoral neck fracture risk with and without inclusion of femoral neck T-scores, drafted a Bland-Altman diagram and calculated Cohen's Kappa coefficient

Key words: FRAX, Fracture probability, Risk assessment, Female, Greece

Address for correspondence:

I. Ilias, 2 E. Venizelou Sq, Athens, GR-11521, Greece; e-mail: iiliasmd@yahoo.com

Received 14-12-11, Revised 03-01-12, Accepted 21-01-12 (with the accepted 3\% 10-year femoral neck fracture risk as a discriminatory threshold). ${ }^{3,4}$

Based on DXA measurements, 17 women had a BMD within normal limits, 53 had osteopenia and 18 had various degrees of osteoporosis. Inspection of the Bland-Altman diagram showed that although the correlation of FRAX scores was acceptable for most women, there were nevertheless inconsistencies for a substantial number of them (Figure 1). Discrepancies in FRAX scores with clinical importance (based on the accepted $3 \%$ 10-year femoral neck fracture risk) were noted in 21/88 women (24\%) (Table 1$)$. The Kappa coefficient was low $(0.33){ }^{4}$

The correlation of femoral neck fracture risk in Greek women using the Italian version of FRAX with

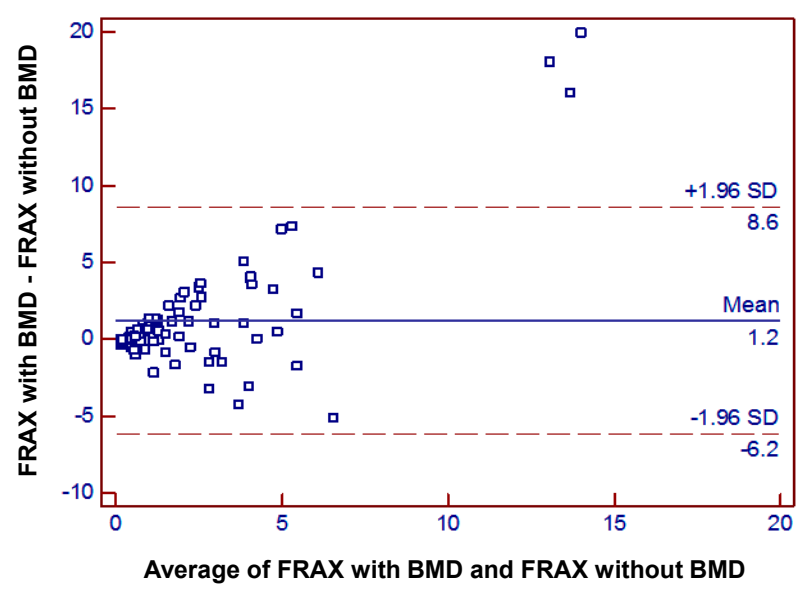

Figure 1. Bland-Altman plot of FRAX estimated 10-year hip fracture rate with and without the inclusion of BMD results. 
Table 1. 2x2 table for calculation of Cohen's Kappa

\begin{tabular}{lccc}
\hline & & $\begin{array}{c}\text { Estimated 10-year hip } \\
\text { fracture rate from } \\
\text { FRAX with BMD }\end{array}$ \\
\hline Estimated 10-year hip & $<3 \%$ & 57 & $\geq 3 \%$ \\
fracture rate from & $\geq 3 \%$ & 7 & 14 \\
FRAX without BMD & $<3 \%$ & 10 \\
\hline
\end{tabular}

and without the inclusion of femoral neck T-scores was not satisfactory $(76 \%)$ and was worse than that reported during the validation of FRAX in other countries (approximately 85\%-90\%). ${ }^{2}$ This finding is important in view of the proposed use of FRAX as a means to identify low- or high-fracture risk patients who do not require $\mathrm{DXA}^{5}$ and must be taken into consideration when interpreting results in Greek women. A Greek FRAX appears to be necessary.

All the authors declare no conflict of interest.

\section{REFERENCES}

1. Kanis JA, Hans D, Cooper C, et al, 2011 Interpretation and use of FRAX in clinical practice. Osteoporos Int 22: 2395-2411.

2. Assessment of osteoporosis at the primary health care level. Report of a WHO scientific group. World Health Organization Collaborating Center for Metabolic Bone Diseases. Sheffield UK: University of Sheffield, 2007.

3. Bland JM, Altman DG, 1986 Statistical methods for assessing agreement between two methods of clinical measurement. Lancet 327: 307-310.

4. Landis RJ, Koch GG, 1977 The measurement of observer agreement for categorical data. Biometrics 33: 159-174.

5. Leslie WD, Morin S, Lix LM, et al, 2012 Fracture risk assessment without bone density measurement in routine clinical practice. Osteoporos Int 23: 75-85. 